\title{
Evidence for the role of potassium in bone health: results of a systematic review and meta-analysis
}

\author{
H. Lambert ${ }^{1}$, V. Boyd ${ }^{1}$, A. Darling ${ }^{1}$, D. Torgerson ${ }^{2}$, P. Burckhardt ${ }^{3}$, L. Frassetto ${ }^{4}$ and S. Lanham-New ${ }^{1}$ \\ ${ }^{1}$ Nutritional Sciences Division, Faculty of Health and Medical Sciences, University of Surrey, Guildford GU2 7XH, \\ ${ }^{2}$ Centre for Health Economics, University of York, York YO10 5DD, ${ }^{3}$ Clinique Bois-Cerf, Lausanne, Switzerland and \\ ${ }^{4}$ CTSI Clinical Research Centre, San Francisco, USA
}

There have been many recent studies researching the effect of $\mathrm{K}$ on bone health ${ }^{(1)}$. The western diet is acid-generating, and including $\mathrm{K}$-rich foods such as fruit and vegetables in the diet may help counteract the detrimental effect this acid can have on the skeleton. This study aimed to collate the data currently available and conduct a systematic review and meta-analysis on the effects of $\mathrm{K}$ on bone health indices, specifically bone mineral density (BMD), bone turnover markers and Ca excretion.

The following words were entered into Pubmed: 'potassium' and 'citrate', bicarbonate, 'health' 'bone' 'fracture' and 'density' and searched for all cross-sectional, longitudinal and intervention studies. One thousand nine hundred and twenty-two papers were identified. Of the seventy-five applicable studies, twenty-seven were eligible to be included in the systematic review, and eight in the meta-analysis.

For cross-sectional studies, the systematic review showed a positive correlation between $\mathrm{K}$ intake and BMD for all sub-groups combined $\left(r^{2}=0.0033\right)$ and for all sites combined $\left(r^{2}=0.007\right)$. For longitudinal studies, there was a positive correlation between dietary $\mathrm{K}$ intakes and BMD $\left(r^{2}=0.007\right)$. Meta-analysis showed the following significant associations:

\begin{tabular}{lccc}
\hline Variables tested & Mean difference & $95 \%$ CI & $P$ \\
\hline Potassium bicarbonate and & -1.27 & $-2.22,-0.31$ & 0.009 \\
urinary Ca & -38.36 & $-70.24,-6.47$ & 0.02 \\
K and urinary Ca & -3.63 & $-7.06,-0.21$ & 0.04 \\
K and hydroxyproline & -0.48 & $-0.91,-0.05$ & 0.03 \\
K and bone resorption & & & \\
\hline
\end{tabular}

Furthermore, as shown in the Forestplots below, potassium bicarbondate/citrate supplementation was associated with a reduction in bone resorption.

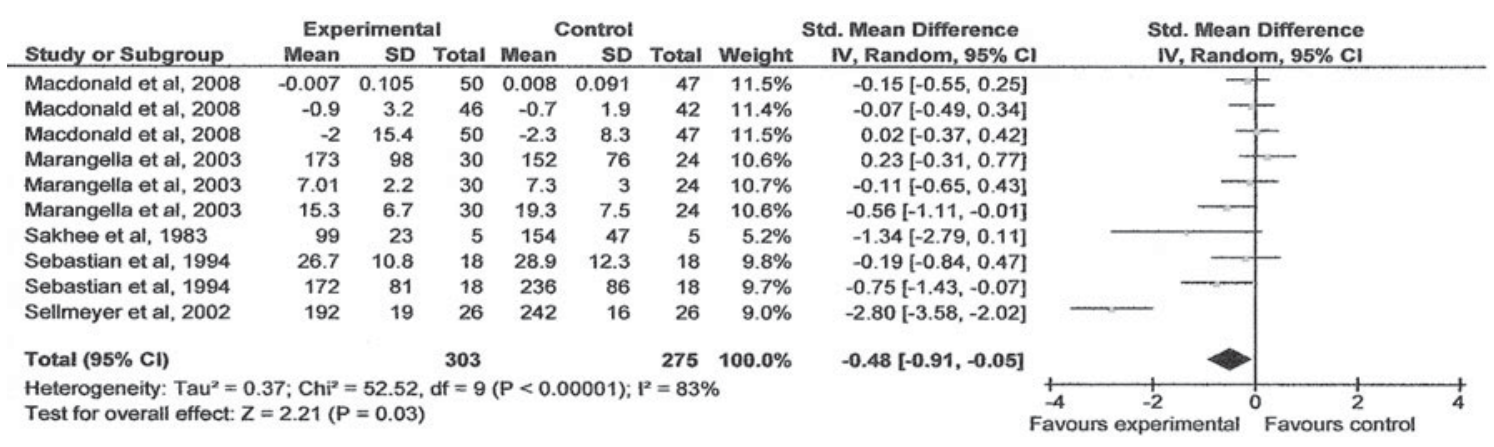

These data suggest that $\mathrm{K}$ has a small $(\sim 1 \%)$ but positive effect on bone. More research is needed into the longer-term effects of $\mathrm{K}$ on fracture risk. However, these preliminary results indicate that dietary $\mathrm{K}$ could be important in the prevention and treatment of osteoporosis.

1. Chan RS, Woo J, Chan DC et al. (2009) Estimates of net endogenous acid production and intake of bone health-related nutrients in Hong Kong Chinese adolescents. Eur J Clin Nutr 63, 505-512. 